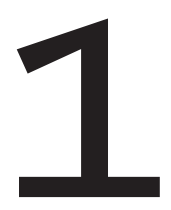

\title{
SOCIEDAD DEL CONOCIMIENTO Y GLOBALIZACIÓN. NUEVOS RETOS PARA LA EDUCACIÓN COMPARADA
}

\section{Knowledge Society and Globalisation. New Challenges for Comparative Education}

\author{
Francesc Raventós* \\ Enric Prats*
}

\section{RESUMEN}

En una época sin apenas referentes y con explicaciones difíciles de relacionar, de comparar y de comprender, nuestros comportamientos y actuaciones se rigen casi exclusivamente por criterios de eficacia o por emociones enérgicas. Es como si al perder los referentes unificadores se produjera una especie de fragmentación en nuestra vida. Las tendencias globalizadoras no han hecho más que aumentar en la última década y tienen que coexistir con el incremento de otras tendencias generadas por las disputas locales y regionales. El artículo aporta algunos elementos de reflexión acerca de la vigencia de la Educación Comparada en este contexto de incertidumbre epistemológica y de hibridación metodológica. Se apuntan algunos elementos que enmarcan la investigación comparativa desde un contexto de globalización y posmodernidad; se revisan algunos

* Universitat de Barcelona. 
de los fundamentos en la investigación comparativa y se apuesta decididamente por la analogía como herramienta de trabajo para conseguir una cierta aproximación en las tendencias descritas anteriormente; y se profundiza en la necesidad de resituar la investigación comparada en un marco tecnológico donde la web 2.0 y las redes sociales han tomado un protagonismo inédito, incluso en las prácticas profesionales y académicas.

PALABRAS CLAVE: Globalización, Posmodernidad, Método Comparativo, Analogía, Tecnologías del Conocimiento, Tecnologías Colaborativas.

\section{ABSTRACT}

In a time when referents are scarce and complex explanations hard to relate to, compare with and learn from, the ways in which we behave and act are governed almost exclusively by our criteria for efficiency or by our emotional impulses. The loss of these unifying referents appears to have made our lives more fragmented. The tendencies towards globalisation have only increased in the last decade and have to coexist alongside an increase in other tendencies that have emerged as a result of local or regional disputes. The article reflects on the validity of some aspects of Comparative Education, in the context of globalisation and postmodernism. Some of the basic tenets upon which comparative research is based are revised and it clearly supports the use of analogy as a tool to be employed when trying to explain to the tendencies described earlier; it analyses in greater depth the need to revise the comparative research within a technological framework whereby the web 2.0 and the social networking sites are now the indisputable protagonists, in both the professional and academic arenas.

KEY WORDS: Globalisation, Postmodernity, Comparative Method, Analogy, Knowledge Technologies, Collaborative Technologies.

$$
* * * * *
$$

\section{INTRODUCCIÓN}

Nos hemos adentrado sin un proyecto común en un período llamado posmodernidad que se caracteriza por la ausencia de un plan, de un rumbo al que dirigirse. Vivimos en una época sin apenas referentes y con explicaciones 
difíciles de relacionar, de comparar y de comprender. Los teóricos de la posmodernidad nos indican que las vidas de las personas han dejado de sentirse aprobadas o legitimadas por algún criterio unificador. Parece como si ante la falta de una fundamentación comúnmente aceptada, cada vez más nuestros comportamientos y nuestras actuaciones se rigen casi exclusivamente por criterios de eficacia o por emociones enérgicas. Es como si al perder los referentes unificadores se produjera una especie de fragmentación en nuestra vida que nos conduce a proceder con criterios dispares, e incluso a veces contradictorios, en cada uno de nuestros ámbitos de actuación.

La mundialización se ha impuesto en todos los campos y afecta a todos los ámbitos. La investigación científica y las múltiples aplicaciones tecnológicas han posibilitado en las últimas décadas un enorme crecimiento económico, y por supuesto tecnológico, en algunos escenarios de nuestro mundo. Las tendencias globalizadoras no han hecho más que aumentar en la última década y tienen que coexistir con el incremento de otras tendencias generadas por las disputas locales y regionales.

El período conflictivo en el que vivimos se nos presenta como un debate continuo de las nuevas realidades sociales y políticas que demandan soluciones inéditas y originales. Y los problemas de siempre adquieren en este momento, gracias a los progresos técnicos, nuevas fisonomías. El enorme desarrollo de las tecnologías nos ofrece ahora grandes posibilidades, que evidentemente resultan tan útiles para construir como también para destruir. En el mundo sin centro que ya anunciaba Nietzsche y que caracteriza a la posmodernidad, no hay apenas espacio para el reposo, todo es urgente para construir una nueva manera de actuar como seres humanos.

No obstante, como indicó Rubio Carracedo, la crítica de la posmodernidad

«persistirá, pese a sus frecuentes exageraciones, porque es básicamente certera; pero su consecuencialidad es la rectificación o el replanteamiento del proyecto ilustrado, nunca su rechazo global, dado que su misma crítica se realiza desde dentro y con las herramientas facilitadas por el programa moderno» (RUBIO CARRACEDO, 1996: 109).

Este artículo pretende aportar algunos elementos de reflexión acerca de la vigencia de la Educación Comparada en este contexto de incertidumbre 
epistemológica y de hibridación metodológica. Se organiza en tres apartados donde, en el primero, se apuntan algunos de los elementos que enmarcan la investigación comparativa desde un contexto de globalización y posmodernidad; en el segundo, se revisan algunos de los fundamentos en la investigación comparativa y se apuesta decididamente por la analogía como herramienta de trabajo para conseguir una cierta aproximación en las tendencias casi opuestas descritas anteriormente; y se profundiza, ya en el tercer apartado, en la necesidad de resituar la investigación comparada en un marco tecnológico donde la web 2.0 y las redes sociales han tomado un protagonismo inédito incluso en las prácticas y las rutinas profesionales y académicas.

\section{LA EDUCACIÓN COMPARADA EN LA GLOBALIZACIÓN Y LA POSMODERNIDAD}

En el debate académico actual suele ser habitual el uso de expresiones y términos que entremezclan, a sabiendas o por repetición de automatismos intelectuales, conceptos que en su origen son ciertamente incompatibles y que se refieren a campos semánticos que en apariencia podríamos considerar algo alejados. Sin duda, las dos palabras que unimos en este artículo, «globalización» y «posmodernidad» remiten a consideraciones culturales y, por supuesto, pedagógicas de distinta índole aunque, como se verá, existe un marcado proceso de ensamblaje de ambos términos que nos puede conducir bien a una absoluta desorientación conceptual y metodológica, bien a una renovación de los supuestos que deben presidir la actividad comparada en educación (GARCÍA RUIZ, 2012). Sin anticipar nada, nos queremos inclinar por el segundo horizonte y ofrecer, en este artículo, algunas trazas de los distintos caminos que podemos ensayar para avanzar en este frondoso y espeso bosque.

Los diagnósticos acerca de las repercusiones de la globalización y de la posmodernidad en la investigación educativa y, en particular, en la educación comparada han proliferado en la última década (WELCH, 2001; BRAY, 2002; COWEN, 2006; MARTÍNEZ USARRALDE, 2006; ASTIZ, 2011). Si bien esconden conceptos que remiten a aspectos bien distintos de la sociedad actual, las nociones de globalización y de posmodernidad se usan como 
pareja indisociable para expresar un cierto estado de estupefacción y de vigilancia, tanto epistemológica como de método, que nos obliga a revisar los fundamentos y procedimientos, pero también la agenda de investigación y los enfoques de trabajo.

Aunque como «globalismo» hemos acuñado uno de los principios rectores para muchas corrientes teóricas en educación —antecedente de la «interdisciplinariedad» y este de la «transversalidad»-, que deseaban superar el encorsetamiento de las disciplinas, ahora nos vemos abocados a emplear el término «globalización» para referirnos al proceso de convergencia a escala mundial en lo económico y financiero, apoyado en la interconectividad que ha favorecido el avance de las tecnologías, y que tiende sus brazos hacia los terrenos político, militar y, por supuesto, cultural, donde la educación quedaría enmarcada. Y todo ello con unas importantes consecuencias en el ámbito de la investigación.

Por su parte, la posmodernidad casi nos insinúa y revela lo contrario. En su formulación primera, el proceso posterior a la modernización de las sociedades iría acompañado de un decaimiento de los fundamentos de la modernidad ilustrada, con pretensión de universalidad, arropados en la razón. Como bien expresaron los autores de la Escuela de Frankfurt, la razón instrumental derivó en un uso aparentemente irracional de los avances científicos, poniendo los efectos de las guerras mundiales del siglo veinte y, por supuesto, de la barbarie nazi, como los ejemplos más emblemáticos.

Pero esa crítica de la razón instrumental derivaría, con los posestructuralistas, en una reclamación de lo emocional como eje articulatorio de las demandas, en aras de una sociedad más equitativa, más tolerante y más acogedora. La unión de ambos hilos, el de la teoría crítica neo-marxista y el de las corrientes posestructuralistas, nos conduciría al punto en el que nos encontramos, donde se han cercenado las bases de los valores absolutos o perennes y se han amplificado notablemente los argumentos para las propuestas relativistas.

En ese intervalo de tiempo, y para inclinar el debate, aparecen los razonamientos y principios de la complejidad, de la sociedad líquida, de la «ciberciudadanía», entre algunos de ellos, que terminan por situar el discurso pedagógico en un punto sin retorno: la aceptación de la autonomía del sujeto, como una reclamación central de la racionalidad moderna, al mismo 
tiempo que una aprobación implícita de las emociones como mecanismo regulador de las relaciones personales en una sociedad posmoderna. Todo ello, por supuesto, muy alejado de cualquier anhelo de carácter universalista $\mathrm{y}$, por ende, global.

Curiosamente la paradoja se produce en la combinación de los dos términos, que un uso indiscriminado o poco crítico puede fácilmente generar confusión o desorientación en la actividad académica, o inclusive ambas cosas al mismo tiempo. Los interrogantes se suceden uno tras otro y la investigación tiene que responder, sin demora, a todos ellos: ¿existe la posmodernidad?, ¿cómo se manifiesta en el terreno educativo y en el pensamiento pedagógico? Es en este supuesto que se discute acerca de la posibilidad misma de hacer pedagogía desde la posmodernidad (AYUSTE y TRILLA, 2005), o lo que es lo mismo, de la existencia de una pedagogía posmoderna, lo cual llevará a interrogarse sobre el encaje de la comparación en un mundo sin referentes y de la duda epistemológica, ya no sólo metodológica, de acometer estudios comparados.

En suma, este período de posmodernidad, donde pierden vigencia los fundamentos de toda índole, «posfundacional» como dirían algunos autores, (NINNES y METHA, 2004; citados por MARTÍNEZ USARRALDE, 2006: 85), distinto de esos otros diagnósticos sobre la «modernidad tardía» o la «modernidad líquida», impide dibujar una idea de finalidad u objetivo para alcanzar en nuestra sociedad, y por lo tanto a una anulación absoluta del concepto mismo de desarrollo y progreso (TAGUIEFF, 2004).

Este grado elevado de relativismo, con la disolución de fronteras epistemológicas y la fragmentación o especialización del conocimiento, nos obliga a repensar la educación y construir la pedagogía desde otros parámetros. En clave educativa, la ampliación del concepto de educación y el reconocimiento de otros agentes educativos, la educación más allá de la escuela o la educación a lo largo de la vida, lo que se está denominando ya «educación expandida», «invisible»y «abstracta», son todas ellas expresiones que extienden y desenvuelven el concepto de educación. La dependencia respecto de la política y evidentemente de los flujos económicos (la «transitología», en palabras de Cowen, recogido por USARRALDE, 2006: 87), conduce a un replanteamiento de la función motora del cambio social por parte de la educación, y también a una revisión de las 
investigaciones educativas de los últimos años, que se han caracterizado por una pérdida de contenido «moral» y de una apuesta ideológica a favor de estudios y análisis de corte empirista o experimental, supuestamente neutrales en valores, lo cual era sólo una pretensión.

Vivimos, en definitiva, pues en un período sugestivo con ricos y variados atractivos que a menudo van acompañados por la incertidumbre y la intriga. $\mathrm{Y}$ en estos tiempos de grandes cambios y de importantes transformaciones tecnológicas, la Educación Comparada transita por nuestra geografía, quizás sin brújula y con falta de guía u orientación, pero con fecundidad y desarrollándose de manera prolífica.

La Educación Comparada que se nos dibuja en el horizonte inmediato deberá, además de examinar corrientes educativas y comparar sistemas educativos o libros de texto, espacios escolares, modelos de financiación educativa y otros recursos, ocuparse también de todos los instrumentos y las tecnologías útiles, más allá de la lógica fundamentada casi únicamente en los manuales y libros de texto.

\section{CUESTIONES DE FUNDAMENTACIÓN DEL MÉTODO COMPARATIVO EN EDUCACIÓN}

El extraordinario desarrollo de la investigación aplicada en el ámbito educativo no debería significar bajo ningún concepto desentenderse y dejar de prestar atención a la investigación básica y metodológica y, por lo tanto, infravalorar aquello que es sustancial o fundamental; es decir, la analogía.

El auge irrefutable del uso de la comparación como procedimiento de análisis conlleva siempre una invitación a reflexionar sobre la metodología comparativa. La enorme proliferación de estudios internacionales en los que lo sustancial y significativo es el mayor conocimiento del «otro» para optimizar o perfeccionar lo propio, es mucho más que una moda casual o una tendencia pasajera. Además, el poder e influjo de los organismos internacionales, y en general de las organizaciones transnacionales, se ha incrementado de forma exponencial a lo largo de las últimas décadas y singularmente desde el final de la Segunda Guerra Mundial. 
La educación actual nos impone una cultura de cambio constante sobre todos los sistemas educativos, sobre las reformas de la educación y sobre el diseño y articulación de las nuevas políticas educativas. Infravalorar el papel determinante, aunque a veces encubierto, de los organismos internacionales podría conducir a una visión de mentalidad provinciana, en el sentido más peyorativo de esta expresión.

En época de globalización, los especialistas en Educación Comparada, y en general los comparatistas, no podemos cerrar fronteras ni acotar un territorio como propio y exclusivo. Por lo tanto, no parece en modo alguno aconsejable instalar ningún tipo de aduana epistemológica; por el contrario, las nuevas demandas de comparación y el uso cada vez más extendido en el ámbito internacional, por ejemplo en la propia OCDE, sugieren que se abran de par en par las fronteras de nuestra disciplina y de nuestras investigaciones. Con ello contribuiremos a alcanzar un nivel de madurez más elevado y de este modo poder afrontar los retos venideros.

Dentro del ámbito de las ciencias sociales, el uso de la metodología comparativa no ha hecho más que aumentar de forma ininterrumpida desde finales del siglo XX. No obstante, llama poderosamente la atención el hecho de que amparados en una malentendida visión exclusivamente praxiológica o pragmática - de puro practicismo que obstruye la reflexión y la creatividad-y por supuesto reduccionista, la mayor parte de los abundantes estudios comparativos adolecen de un análisis riguroso y científico en torno al método. Es decir, utilizamos cada vez más el método comparativo sin preocuparnos suficientemente de su fundamentación, de su validez y de su utilidad.

Ordenar los fenómenos y generalizar suelen ser dos objetivos explícitos o no- que acompañan a la formulación de hipótesis. Pero podemos cuestionarnos si es la generalización la que precede a la comparación, o bien si es la comparación la que origina la generalización. Por un lado, podríamos afirmar que el objetivo de cualquier tipo de comparación es la generalización; pero además, toda generalización necesita verificar su validez y fundamentarse en estudios comparativos acerca de la naturaleza y las causas de los fenómenos.

Claude Levi-Strauss, en su Anthropologie structurale, defiende que «no es la comparación la que motiva la generalización, sino al contrario» (LÉVI- 
STRAUSS, 1958: 28). No resulta sencillo sostener hoy tal afirmación sin establecer matizaciones. De hecho toda investigación tiende o debería tender a algún tipo de generalización. Mientras «la génesis o raíz de la comparación tiene un carácter eminentemente diversificador, el fruto o resultado esperado de la investigación comparativa es más bien de índole unificador» (RAVENTÓS, 1990: 110). Y como indica Lê Thành Khôi, no es imposible generalizar a partir de casos particulares: «une analyse approfondie peut y conduire en suscitant des hypothèses d ordre général, alors qu une comparaison superficielle de nombreux systèmes n'y parvient pas» (LÊ THÀNH KHÔI, 1981: 33).

La incorporación de la Educación Comparada al ámbito de las ciencias sociales, de forma crítica, es la mejor garantía de su progreso futuro. Prosperar, mejorar o perfeccionar nuestra disciplina equivale a que las investigaciones en Educación Comparada no se realicen al azar y sin una cierta sistematización. Recordemos a Pedro Rosselló cuando hace ya casi medio siglo nos indicaba que la mayor parte de las investigaciones en educación comparada no desarrollaban de hecho ningún tipo de comparación y se acostumbraba a delegar en el lector la responsabilidad de descubrir las correspondencias y las diferencias (ROSSELLÓ, 1963).

En realidad, no fue hasta después de Bereday que los problemas metodológicos de la comparación empiezan a estudiarse con cierta profundidad. Parecía como si no fuera preciso conocer la esencia de la comparación y tampoco definir el concepto metodológico de la comparación. Era suficiente determinar un tercer término de la comparación, un referente «respecto a», es decir, un tertium comparationis. Y comparar científicamente no puede ser tarea fácil en la medida que el propio concepto de comparación presenta varias dimensiones y problemas.

Aunque gocemos del amparo y protección de las ciencias sociales, estas disciplinas tienen pocos modelos y discursos teóricos que sean realmente incuestionables, a pesar de que han logrado recopilar abundante información y muchos datos empíricos. Nuestro afán por resolver problemas prácticos inmediatos y por evitar los posibles conflictos ocasionados por la crítica puede dirigirnos a ignorar, o a no prestar la suficiente atención, a la importancia de las cuestiones metodológicas. Es decir, falta crítica y reflexión en los comparatistas actuales. 


\subsection{La Analogía: Investigar el Fundamento}

En las dos últimas décadas, el enorme proceso de expansión y de diversificación de titulaciones en distintas universidades europeas, y sobre todo y singularmente en España, debería ser objeto de una seria reflexión. Junto a indiscutibles aspectos positivos de modernización, hallamos olvidos y dispersiones. Uno de los efectos negativos de estos procesos de expansión y de diversificación está relacionado con una cierta «pérdida de identidad» y con una desconexión cada vez más frecuente de las disciplinas filosóficas (Lógica, Metafísica, Ética, Epistemología...), sustento teórico y metodológico que debería ayudarnos a conocer y comprender el razonamiento analógico como fundamento de la metodología comparativa y de la analogía como método científico.

Conviene recordar que todos los métodos comparativos proceden de la analogía, y que el razonamiento analógico implica y se fundamenta en el razonamiento deductivo-inductivo o bien inductivo-deductivo. El estudio de analogías y simetrías — biológicas, sociales, jurídicas, del lenguaje, del pensamiento, etc.- , su reflexión y profundización, generaría sin duda un efecto beneficioso en la correcta utilización de la metodología comparativa.

En el campo de la investigación científica y especialmente en ciencias sociales, la comparación no cumple únicamente una función de aplicación general, sino que permite una amplia aplicación metodológica de sumo interés para el desarrollo de sus disciplinas, y que podemos denominar como métodos comparativos, o en un sentido unitario que los engloba a todos ellos: método comparativo.

Siguiendo el enfoque del maestro Alejandro Sanvisens ${ }^{1}$, podríamos distinguir dos acepciones o sentidos: uno amplio y de carácter general, según el cual englobamos la comparación como un razonamiento lógico de gran amplitud relativo a la mayoría de las actividades intelectuales humanas; y otro más estricto en el que el método comparativo se presenta como un procedimiento ordenado y sistemático que relaciona fenómenos diversos, con

${ }^{1}$ Sería interesante recuperar el excelente artículo de Alejandro SANVISENS (1973): «El enfoque sistémico en la metodología educativa. (La educación como sistema)». En Reforma cualitativa de la educación. Madrid, Sociedad Española de Pedagogía (Instituto de Pedagogía del CSIC), pp. 245-275. 
objeto de descubrir las semejanzas, las diferencias y las relaciones entre los distintos sujetos o elementos de la comparación.

El estudio de la analogía entendida en un sentido amplio como relación de semejanza o correspondencia, y sus posibilidades como procedimiento y como razonamiento científico, fundamentan y dan sentido al análisis comparativo propio de la Educación Comparada. Como ya hemos indicado, es evidente que el estudio de estos fundamentos que son comunes a diversas disciplinas procede mayoritariamente de trabajos ajenos a la Educación Comparada. En este sentido, insistimos en que los comparatistas de la educación han efectuado muchas aportaciones en favor de lo educativo, y pocas en favor del estudio de la analogía y del método comparativo.

Por otro lado, sabemos que la analogía y el razonamiento analógico intervienen en la mayor parte de los procesos de aprendizaje. Por ello, desde los más elementales juegos educativos diseñados para niños y niñas de muy corta edad, hasta los rompecabezas, juegos de construcción, materiales didácticos, etc., están fundamentados en procesos de aprendizaje basados en correspondencias, imitaciones, asociaciones, etc.; con una lógica interna equivalente a la propia de la metodología comparativa. Piénsese en la cantidad de problemas, ejercicios o actividades propuestas en los libros de texto y materiales pedagógicos de cualquier nivel educativo, desde la educación infantil hasta la educación superior, en los que la proporcionalidad, la semejanza, la atribución o atributo, la metáfora, etc., juegan un papel esencial, por ejemplo en los procesos de aprendizaje de las matemáticas y de las lenguas, tanto propias como extranjeras.

La persistente utilización de las metáforas, tropos, lenguajes figurados y otras ficciones alegóricas, tan frecuentes en nuestro mundo mediático y tecnológico nos invita a reflexionar seriamente sobre el papel y el sentido de la comparación ante los desafíos que se nos avecinan en los próximos años. En la medida en que seamos capaces de descubrir el valor formativo de la analogía y del método comparativo en muchos de los procesos de aprendizaje, tanto escolares como extraescolares, estaremos también contribuyendo a una mayor y mejor profundización metodológica.

Veamos una muestra concreta a partir, por ejemplo, de una analogía biológica entre la circulación sanguínea y la asistencia a la escuela para aprender. Sabemos que las células de la sangre se cargan de oxígeno y lo 
transportan por el cuerpo de manera análoga a los alumnos que acuden al centro educativo para aprender y luego regresan a su domicilio. El recorrido de los escolares - la asistencia a la escuela para aprender- es pues, un fenómeno análogo a lo que sucede con los glóbulos rojos que se desplazan a los pulmones para recargarse de oxígeno y luego poder transportarlo a las células. O la analogía entre los nutrientes con que nos alimentamos - y que nos aportan energía - y, por ejemplo, el combustible de un vehículo —energía producida por la combustión del carburante— que nos sirve para desplazarnos. Sabemos que muchas ideas no se unen por casualidad, sino que existen determinados principios de conexión entre ellas, ya sea por una relación de semejanza, de espacio o tiempo contiguo, de causa o de efecto, etc.

Igualmente podríamos sugerir diversas muestras de analogías, ejemplificadas en el campo de la Química o la Física — con los estados de la materia, el calor, la densidad, los átomos y moléculas, etc.- en el ámbito del lenguaje (frases, palabras, letras...), en el terreno del conocimiento científico, en el dominio de la acción social, y por supuesto en el campo del sistema educativo. Como señalaba en sus clases el filósofo y académico Emilio Lledó, las metáforas no son solamente una forma de describir o de pronosticar, sino que muchas veces son un motor para transformar el mundo. Por ello, en la sociedad del conocimiento y de la globalización necesitamos conocer el poder del lenguaje y descubrir las distintas metáforas que podemos aplicar a Internet y a las nuevas tecnologías, como sería el caso, por ejemplo, del concepto de «autopistas de la información», aunque sin peaje de momento- en el caso de Internet.

Sin lugar a dudas, y a lo largo de toda la historia de la humanidad, la analogía y las metáforas nos resultan útiles para iluminar nuestros conocimientos, además de ayudarnos a comprender mejor el mundo que nos rodea, la sociedad en que vivimos. Recordemos que Platón ya establecía analogías entre el individuo y la ciudad o entre Dios y el Sol, como la luz que nos permite iluminar nuestro conocimiento. La analogía sirve para descubrir el quid —el intríngulis - de la cuestión, para profundizar en aquello que parece oculto. Es un instrumento metodológico productivo que resulta eficaz y valioso en diferentes ámbitos del pensamiento. También la analogía se aprovecha para comprender, para desentrañar el sentido y así poder escudriñar en lo recóndito y oculto, para acceder a un sentido más profundo de un 
concepto. Es decir, a través del razonamiento analógico podemos facilitar la comprensión de un concepto y penetrar en su sentido más profundo.

En este tiempo actual de grandes progresos y enorme desarrollo de las tecnologías y de la globalización, la Educación Comparada precisa profundizar en las limitaciones, aplicaciones y beneficios de la analogía y del pensamiento comparativo. Y deberemos reubicar otra vez las investigaciones comparativas en el naciente marco tecnológico.

\section{LAS TECNOLOGÍAS DEL CONOCIMIENTO Y SU IMPACTO EN LA INVESTIGACIÓN COMPARADA}

La descripción llevada a cabo en las páginas precedentes acerca del contexto de posmodernidad y globalización y sus repercusiones en la metodología comparativa, exige atender en último término a los retos que plantea la sociedad del conocimiento y, más en concreto, acerca del papel que están asumiendo las nuevas tecnologías. En este análisis final, convendrá destacar también el desarrollo experimentado por la educación en las últimas décadas, tanto en extensión como en intensidad, unido a la implantación de las tecnologías digitales en casi todos los rincones de la vida cotidiana, lo que en la práctica está suponiendo una transformación importante en los hábitos investigadores. Hacer pedagogía en clave comparada, en este entorno de alta sofisticación tecnológica, obliga no solo a revisar las metodologías al uso, sino también a mirar con otras ópticas de qué manera este entorno modifica la misma generación de conocimiento.

Como afirma Schriewer, las tendencias globalizadoras corren el riesgo de perder de vista las singularidades particulares de las realidades próximas, unas realidades que han sido el objeto de trabajo tradicional de las ciencias sociales: «Las nuevas concepciones que tienden crecientemente a ver la realidad de las sociedades modernas como una única sociedad global, tienen como consecuencia la disolución del objeto de conocimiento de las ciencias sociales comparadas» (SCHRIEWER, 2000: 32).

Así, un mundo que había crecido bajo el paradigma moderno de los Estados-nación, cada uno con sus respectivas diferencias y particularidades nacionales —entendidas como propias y originales-, se está viendo 
abocado precisamente a una disolución de esas diferencias, convertidas ahora en desigualdades, lo que aleja a la educación comparada de su anhelado objetivo programático de establecer modelos de referencia, según un determinado tertium comparationis, en la microescala para acercarse definitivamente a los estudios de carácter global. Como explica el profesor Leoncio Vega:

«La globalización y la educación comparada parecen, en principio, irreconciliables por su distinto carácter y naturaleza; la globalización conlleva la supresión del Estado-nación y la homogeneización mundial. Por el contrario, la educación comparada se fundamenta en los sistemas educativos y estos se circunscriben al Estado-nación, que es el que los establece y dirige» (VEGA, 2001: 86).

Las etnografías nacionales, con una presencia notable en los catálogos de revistas comparatistas, tienden a relajar su objetivo último, de ofrecer esquemas de reconocimiento para reflejar el grado de acercamiento a corrientes globales, y se orientan sobre manera a buscar el exotismo de la diferencia, a indagar lo que de original y propio presenta la nación o cultura estudiada.

Se trata, en un contexto intelectual sometido al giro posmoderno, de cambiar el mecanismo con el que miramos a la educación. En este sentido, insiste Paulston que la posmodernidad, siguiendo a Lyotard, nos increpa justamente a mirar con la mirada o, en sus palabras, que «la sensibilidad posmoderna es principalmente visual y rompe esta colonización del inconsciente mediante el discurso verbal» (PAULSTON, 2000: 260).

En este sentido, la explosión de las diferencias remite a una consideración epistemológica pero también metodológica: más allá de la realización de amplios catálogos de las diferencias, lo que puede imprimir un nuevo enfoque a la Educación Comparada es el dibujo de cartografías, a modo de mapas de carreteras, donde se señalan los puntos de interés y los mojones que indican la situación en cada momento. Por supuesto, se trata de cartografiar para informar de las fronteras y de los límites, además de enfatizar la diferencia como motor del cambio social.

Como indica Paulston, «mientras que los textos de los geógrafos modernos suelen representar el espacio como un lugar inocente de objetos 
situados dentro de fronteras fijadas, coordenadas y esencias, los textos de los cartógrafos posmodernos suelen presentar un espacio agonista y controvertido de lugares y fronteras que cambian continuamente» (PAULSTON, 2000: 270). En realidad, lo que plantea Paulston, además de ese giro en la mirada, es una apuesta para que esa mirada sea al mismo tiempo integrada e integradora, interdisciplinaria y global, lo que en tiempos de tecnologías sociales y colaborativas se aventura mucho más sencillo.

Por supuesto, esta analogía que hemos ensayado con los mapas de carreteras no es gratuita ya que en la comparación de la posmodernidad se tratará de transitar por caminos ya conocidos pero no descubiertos a la luz de las nuevas sensibilidades que exige ese nuevo enfoque de las diferencias. Las tecnologías sociales, que ponen el énfasis en lo colaborativo, aportarán una nueva capa en esta cartografía.

\section{LA IMPLOSIÓN DE LAS TECNOLOGÍAS COLABORATIVAS: EDUCACIÓN COMPARADA EN LA WEB 2.0}

Aquí, la clásica división entre investigación cuantitativa e investigación cualitativa, decantadas desde siempre hacia objetivos nomotéticos o ideográficos, respectivamente, se está diluyendo gracias al desarrollo de los sofisticados artefactos tecnológicos. El sueño de los comparatistas de hace un siglo, de acceder a ingentes cantidades de datos, se está cumpliendo al mismo ritmo que aumenta el desencanto por la ingobernabilidad de tanto material en bruto. Proliferan los informes de segundo nivel, que aportan información depurada a partir de datos no recopilados por el mismo investigador, e incluso de tercer nivel, orientados a la interpretación de los informes del nivel anterior. Parecería que se funde aquella clásica división que comentábamos, en el sentido de que lo cuantitativo, una vez reconocida la imposibilidad nomotética de alcanzar leyes universales (GARCÍA GARRIDO, 1986, p. 136), más aún mediante la acumulación de datos, se hibrida con lo cualitativo, que «adopta tanto un enfoque ideográfico como nemotético, localizando sus resultados en períodos específicos de espacio y tiempo» (FAIRBROTHER, 2007, p. 42).

En nuestros días, la investigación científica tiene un marcado acento cooperativo y su legitimidad proviene, además del supuesto rigor y 
sistematicidad, de su interés por la difusión abierta que permite el intercambio y la discusión sobre hallazgos y avances. Por supuesto, el científico social no es inmune a las influencias de su entorno, que está siendo definido por una fuerte tendencia hacia el individualismo y la competitividad en su vertiente más perversa. Sin embargo, como señalan Ortega y Rodríguez, la misma peculiaridad científica nos impide alejarnos

«del espíritu colaborativo que la funda: son solamente los pares, los integrantes de la comunidad científica de la que el evaluado forma parte, quienes atribuyen y reparten la especie específica de capital simbólico que se maneja como divisa corriente dentro de ese campo bien delimitado, capital específico que puede o no traducirse, transformarse, en otro tipo de capitales (monetario, por ejemplo)» (ORTEGA \& RODRÍGUEZ, p. 80; la cursiva es nuestra).

Fuera del ámbito académico, por ejemplo, resulta en la práctica muy difícil de entender cuáles son los mecanismos y procesos, con sus repetidas acreditaciones y evaluaciones externas - necesarios para conseguir tramos, plazas, publicar artículos, o presentar ponencias y comunicaciones a congresos, etc.- - que se esconden en la producción y difusión de la ciencia. La clave de todo ello no radica precisamente en las ganancias económicas que conlleva la actividad académica, sino en el prestigio, o «capital simbólico», como expresa la cita anterior, de corte intelectual (este sí, acumulable), que acarrea el reconocimiento por parte de los iguales. Esta característica genuinamente científica, junto con la inquietud por la difusión e impacto de su actividad, fueron las bases precursoras de la World Wide Web, puesta a disposición del público, a partir de 1991, por Tim Berners-Lee, del CERN de Ginebra, quien se limitó a implantar la modalidad de hipertexto en Internet (creada en 1969, en la Universidad de California de Los Ángeles). La web 1.0, como ahora la conocemos, supuso un cambio trascendental en los procedimientos de investigación científica, por su evidente capacidad para el intercambio y la inmediatez en la recuperación de información, entre otras ventajas, pero sobre todo porque abrió la posibilidad de expandir el conocimiento a más y a nuevos públicos.

Encerrados en las revistas de impacto, en su versión impresa, la localización de esos hallazgos no dejaba de ser costosa y prolongada, siempre a cargo de profesionales del sector, documentalistas, o por los 
mismos investigadores. La supuesta torre de marfil, que encierra a una comunidad científica alejada de la sociedad, se está convirtiendo en una torre de cristal, cuya transparencia empieza a modificar los hábitos de trabajo de muchos investigadores. Esta transformación viene de la mano de la Web social y colaborativa. Nos referimos al papel que está ejerciendo la tecnología Web 2.0, con su producto estrella, la fórmula wiki, que se erige como emblema de lo que ha venido a denominarse como procomún o conocimiento compartido.

En realidad, por su origen y desarrollo, la Web se convierte en un reflejo de la evolución de la producción científica. De las primeras andanadas, más centradas en el acceso rápido e hipertextual a la información, hasta los productos actuales, que permiten la creación compartida de conocimiento, la comunidad científica ha visto cómo se incrementaban también los filtros para la difusión de sus trabajos. Así, por ejemplo, los mecanismos de evaluación del impacto científico — creados para rentabilizar la financiación de la administración norteamericana en los costosos proyectos de investigación de los sesenta- han encumbrado una filosofía de trabajo, ciertamente competitiva e individualista, que se da de bruces tanto con el mismo espíritu científico como con la filosofía abierta de la Web.

A estas alturas, empieza a ser difícil poder defender que el impacto de un hallazgo científico se pueda medir por las veces que ha sido citado ese mismo hallazgo en otros textos científicos. La torre de cristal no admite esa contabilidad añeja: «El surgimiento de la Web entraña, precisamente, la emancipación de los científicos respecto a los canales tradicionales de intermediación».

En realidad, como venimos siendo testigos repetidamente, no admite discusión la repercusión de los informes PISA, no sólo en el plano mediático y político, sino curiosamente también en el campo científico, unos informes que no han seguido los protocolos de la producción científica, lo cual no suele generar discusiones acerca de si les extrae rigor o sistematicidad. Informa, este fenómeno, de cómo unos informes que hace tan solo una década eran material para iniciados o para especialistas del tema, ahora han sucumbido al mercado mediático e incluso están transformando las condiciones del debate ilustrado. 
La Web 2.0 está permitiendo que más usuarios intervengan en este proceso de debate público y, por qué no, de creación de conocimiento. Desde Alvin Toffler, en The third wave (1980), el término prosumer se refiere a ese perfil de usuario que emerge con las tecnologías sociales y colaborativas. El usuario de las primeras tecnologías interactivas añadía a su función de consumidor (consumer) un nuevo rol, el de productor (producer), gracias precisamente a las posibilidades que empezaban a ofrecer los nuevos artefactos en cuanto a la realización y posterior difusión masiva de textos, tanto alfabéticos como audiovisuales. La antigua ilusión de democratizar el conocimiento adquiría una nueva dimensión, además de un empuje sustancial. Por supuesto, la explosión de la primera Web multiplicó, sin sospecharlo inicialmente, los efectos de aquel nuevo rol. En la Web 2.0, la interactividad de la primera Web vino a ser superada por la red social, que admite una cantidad ingente de aportaciones con un esfuerzo insignificante y una preparación técnica mínima. A la rapidez y economía del trabajo en un entorno ofimático, se une la posibilidad de participar en grupos interactivos que, de manera aparentemente desinteresada, contribuyen a producir y difundir conocimiento como nunca antes habíamos sospechado.

El ejemplo paradigmático lo ofrece Wikipedia, una plataforma colaborativa, construida con aportaciones altruistas, que ha venido a subvertir los procedimientos de búsqueda de información no sólo de la gente común sino también de la mayoría aplastante de universitarios, para mal de una buena parte del profesorado, especialmente el de raíz tecnofílica. A más de una década de su creación, la enciclopedia digital contiene más de 20 millones de artículos, a un ritmo diario de más de 11.000 artículos nuevos, y cerca de un millón y medio de colaboradores, a razón de más de 14.000 wiquipedistas nuevos cada día ${ }^{2}$. Por supuesto, esta elevada cifra de usuarios podrá verse incrementada aún más en los próximos años gracias a la extensión de las tecnologías, que se verá favorecida por la implantación de dispositivos y aplicaciones cada vez más sencillas, lo cual redundará en el tráfico de datos que circulará por la red: según el portal Mashable, con datos aportados por Cisco Systems, en 2015 se espera cuadriplicar el tráfico de

2 Para conocer datos actualizados, debe consultarse la página web siguiente: http://stats.wikimedia.org/ES/TablesWikipediansNew.htm (consultado el 27 de diciembre de 2011). 
datos anual en la red, alcanzando la inimaginable cifra de un Zettabyte, el equivalente a los bytes almacenados en 2010 en todos los dispositivos digitales disponibles en el planeta ${ }^{3}$.

De todas maneras, en clave educativa, lo fundamental no es tanto el volumen de información sino el tratamiento que le daremos, para desecharla, transmitirla o generar nuevo conocimiento. Como indica Jenkins, «ninguno de nosotros sabe realmente cómo vivir en esta era de convergencia mediática, inteligencia colectiva y cultura participativa. Estos cambios provocan ansiedades e incertidumbre, incluso pánico» (JENKINS, 2008: 176).

En definitiva, hoy en día el conocimiento se construye gracias a una multiplicidad de plataformas y mediante un número elevado de dispositivos. En cualquier biblioteca pública, resulta sencillo encontrarse con una combinación equilibrada de personas que están consultando al mismo tiempo, y sin una línea definitoria clara, material impreso y material digital, que trabajan con revistas o libros depositados en las estanterías, acompañados de ordenadores o tabletas digitales donde abren portales de información, wikipedias varias, visionando algún documental en YouTube o Vimeo o preguntando en alguna red social, e intentando sintetizar esos insumos en algún editor de textos. Esto empieza ya a ser normal, y no únicamente en las bibliotecas universitarias, sino en las más populares de cualquier pueblo o barrio, para prepararse una oposición, organizar un viaje, planear la compra de un vehículo, o incluso para aprender recetas de cocina o conocer mejor el universo.

Lo que algunos empiezan a denominar la educación expandida, supera y quizás integra lo que en pedagogía habíamos bautizado como educación formal, no formal e informal. El reto para el estudio comparativo de la educación y, por lo tanto, para la Educación Comparada, aumenta exponencialmente con la Web 2.0, que nos obligará a estar atentos acerca de su evolución para no descartar ninguna de sus prestaciones. Si la Web 1.0 vino de la mano de la ciencia y la 2.0 fue una consecuencia lógica de la democratización del conocimiento en la red, las expectativas para la Web 3.0,

${ }^{3}$ Información obtenida en el portal mencionado: http://mashable.com/2011/06/09/globalinternet-traffic-infographic (consultado el 27 de diciembre de 2011). 
que hoy se orientan principalmente hacia el ocio y el entretenimiento, nos conducirán de nuevo a un replanteamiento del objeto y del método.

En nuestros días la Educación Comparada no demanda solamente el trabajo de comparatistas que actúen como pasajeros circunstanciales de nuestra disciplina, sino que precisa además de comparatistas tripulantes que, de modo decidido y práctico, puedan pilotar una nueva educación y que sean líderes o guías, en este mundo posmoderno y globalizado, al que debemos enfrentarnos ya, ahora mismo.

\section{REFERENCIAS BIBLIOGRÁFICAS}

AYUSTE, A. y TRILLA, J. (2005): Pedagogías de la modernidad y discursos postmodernos sobre la educación, Revista de Educación, 336, pp. 219-248.

AZTIZ, M. F. (2011): Los desafíos de la educación comparada contemporánea para informar el debate político-educativo: Una perspectiva teórico-metodológica», Revista Latinoamericana de Educación Comparada, 2, pp. 63-72.

BRAY, M. (2002): Comparative education in the era of globalisation: evolution, missions and roles, Revista Española de Educación Comparada, 8, pp. 115-135.

COWEN, R. (2006): Acting comparatively upon the educational world: puzzles and possibilities, Oxford Review of Education, 32 (5), pp. 561-573.

FAIRBROTHER, F. P. (2007): Quantitative and Qualitative Approaches to Comparative Education, en M. Bray et al. (Eds.), Comparative Education Research: Approaches and Methods (Hong Kong, Comparative Education Research Centre), pp. 39-62.

GARCÍA GARRIDO, J. L. (1986): Fundamentos de Educación Comparada (2ª reimpresión) (Madrid, Dykinson).

GARCÍA RUIZ, M. J. (2012): La universidad postmoderna y la nueva creación de conocimiento, Educación XXI, 15 (1), 179-193.

JENKINS, H. (2008): Convergence Culture. La cultura de la convergencia de los medios de comunicación (Barcelona, Paidós).

LÉVI-STRAUSS, C. (1958): Anthropologie structurale (Paris, Plon).

LÊ THÀNH KHÔI (1981): L éducation comparée (Paris, Armand Colin).

MARTÍNEZ USARRALDE, M. J. (2006): La Educación Comparada revisitada: Revisión a la evolución epistemológica y temática en la era postcomparada, Ten- 
dencias Pedagógicas, 11, pp. 77-100.

ORTEGA, F. y RODRÍGUEZ, J. (2011): El potlatch digital. Wikipedia y el triunfo del procomún y el conocimiento compartido (Madrid, Cátedra).

PAULSTON, R. G. (2000): ¿Un giro espacial en la educación comparada? Construcción de una cartografía social de la diferencia, en J. SCHRIEWER (Comp.), Formación del discurso en educación comparada (Barcelona, Pomares), pp. 259-307.

RAVENTÓS, F. (1990): Metodología comparativa y pedagogía comparada (Barcelona, Boixareu Universitaria).

ROSSELLÓ, P. (1963): Concerning the Structure of Comparative Education, Comparative Education Review, 7 (1), pp. 103-112.

RUBIO CARRACEDO, J. (2000): Educación moral, postmodernidad y democracia (Madrid, Trotta).

SCHRIEWER, J. (2000): Educación comparada: un gran programa ante nuevos desafíos, en J. SCHRIEWER (Comp.) (2002) Formación del discurso en educación comparada (Barcelona, Pomares), pp. 13-38.

TAGUIEFF, P.A. (2004): Le sens du progrès. Une approache historique et philosophique. (Paris, Flammarion).

VEGA, L. (2011): La educación comparada e internacional. Procesos históricos y dinámicas globales (Barcelona, Octaedro).

WELCH, A. (2001): Globalisation, Post-modernity and the State: Comparative education facing the third millennium, Comparative Education, 37 (4), pp. 475-492.

\section{PROFESIOGRAFÍA}

\section{Francesc Raventós Santamaría}

Profesor Titular de Teoría e Historia de la Educación en la Facultad de Pedagogía de la Universitat de Barcelona. Es profesor de Educación Comparada, de Pedagogía Internacional y de Educación y Unión Europea. También es profesor del Máster en Educación para la ciudadanía y en valores y del Máster de investigación en la asignatura sobre Investigación histórica, filosófica y comparativa, en la Universitat de Barcelona. Anteriormente también fue docente en programas de Doctorado sobre Educación y Democracia y sobre Pedagogía Social. Es miembro de la Sociedad Española de Educación Comparada (SEEC). Ha desarrollado 
diversos trabajos de investigación sobre la integración europea en el ámbito de la educación, sobre la centralización y descentralización educativas, sobre reformas de los sistemas educativos en España y en las Comunidades Autónomas, y sobre las políticas sociales y educativas en la Unión Europea. Asesor pedagógico en diversas instituciones públicas y privadas. Ha sido profesor visitante en universidades de Chile, Argentina y Perú. Es autor de diversas publicaciones, entre ellas: Metodología comparativa y Pedagogía comparada. Barcelona, Boixareu Universitaria. Y en colaboración, Los sistemas educativos europeos ¿Crisis o transformación? Barcelona, Fundación «la Caixa» y Educación internacional. Valencia, Tirant lo blanch.

\section{Enric Prats Gil}

Profesor Agregado de Teoría e Historia de la Educación en la Facultad de Pedagogía de la Universitat de Barcelona. Imparte las siguientes materias del Grado de Pedagogía: Educación Comparada; Interculturalidad e Inmigración; Pedagogía de los Medios de Comuncación. Es profesor en el Máster en Educación para la ciudadanía y en valores, en el Máster en Psicopedagogia de la Universidad de Barcelona; en el Máster en Migraciones Contemporáneas, de la Universidad Autónoma de Barcelona, y en el Máster en Educación inclusiva, de la Universidad de Córdoba. Forma parte del Grupo de Investigación en Educación Moral (GREM), de la Universidad de Barcelona, y del Programa de Educación en Valores del ICE de la UB. Es miembro del Comité Ético de la Federación Catalana de ONG para el Desarrollo. Ha publicado Multiculturalismo y educacion para la equidad (Octaedro, 2009), Teoría de la Educación (UOC, 2010) y Diversidad y complejidad en la escuela secundaria (Horsori, 2012).

Datos de contacto: Universitat de Barcelona. Facultat de Pedagogia. Dept. Teoria i Història de l'Educació. Campus Vall d'Hebron. Passeig de la Vall d'Hebron, 171. 08035 Barcelona. Emails: fraventos@ub.edu / enricprats@ub.edu

Fecha de recepción: 23 de enero de 2012

Fecha de revisión: 6 de marzo de 2012 y 7 de marzo de 2012

Fecha de aceptación: 14 de marzo de 2012 